\title{
Descanso postnatal parental: experiencia \\ en lactancia materna de madres trabajadoras \\ usuarias del sistema público de salud en la \\ Región de la Araucanía, de Chile
}

\author{
Parenteral postnatal resting: experience \\ in breast feeding of workers mothers \\ using public health system in \\ Region of Araucania of Chile
}

\begin{abstract}
The prevalence of exclusive breastfeeding up to the sixth month of age does not exceed 60\% in Chile, despite the enactment in 2011 of the Parental Leave Law, which entitles mothers to stay with the newborn up to the twenty-fourth week of life. Given the above, the aim of this study was to explore, from mothers' perspective, which are the aspects involved on the length of the Exclusive Breastfeeding Period. Subjects and method: Qualitative Exploration using a grounded theory approach. Semi-structured interviews and five focus groups were conducted with 35 and 30 participants respectively; therefore a total of 65 working mothers took part in the study. Inductive content analysis was performed using atlas ti-6.2 software package. Validity criteria included triangulation by research and by technique. Results: The elements identified as encouraging breastfeeding are the length of maternal leave and the support of the couple, family and health professionals. Those representing barriers were the presence of breastfeeding-induced pain and early weaning decision, which might be influenced by family or health professionals. Conclusions: Given the strong dependence of factors such as partner and family on breastfeeding maintenance, it is suggested that the involved health professionals shall consider these aspects in promotion.

Key words: breastfeeding; qualitative research; social support; health promotion; health personnel.
\end{abstract}

\section{INTRODUCCIÓN}

Son innumerables las propiedades de la leche humana y del vínculo que se produce entre el recién nacido y su madre al momento del amamantamiento. La lactancia materna contribuye al desarrollo físico y mental del niño, proporcionándole nutrientes en calidad y cantidad adecuadas. Se recomienda la lactancia materna exclusiva (LME) durante los primeros seis meses de vida y su mantención en forma parcial hasta los dos años efectuando un destete respetuoso. Los niños alimentados con leche materna (LM) tienen menor riesgo de contraer enfermedades infecciosas, menor incidencia y severidad de éstas, menor probabilidad de desarrollar sobrepeso, hipertensión y
Bernardita Baeza W. $(1,2,3)$

Fabiola Henríquez K. (1)

Ruth Prieto G. $(1,3)$

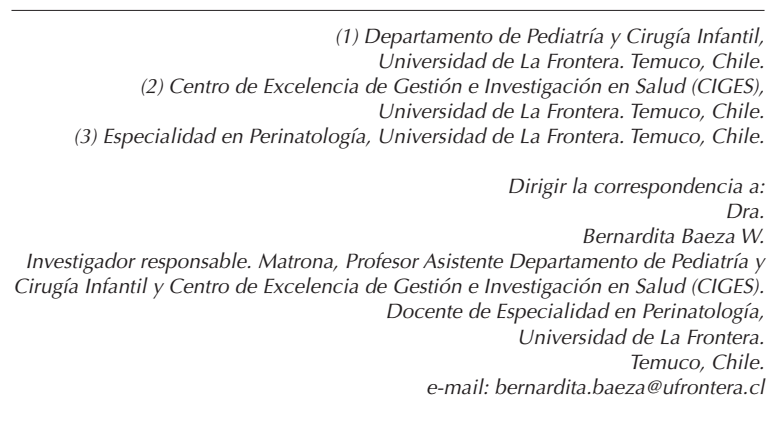

Este trabajo fue recibido el 23 de Noviembre de 2015 y aceptado para ser publicado el 3 de Marzo de 2016.

diabetes mellitus a largo plazo (1). La LME determina un menor gasto de recursos en hospitalización, medicamentos y atención profesional(1). Además, los niños alimentados los primeros seis meses de vida con $L M$ demuestran mejor desempeño en test de inteligencia (2-7) y al tener una mejor interacción con la madre, son más seguros y estables $(8,9)$. Asimismo, altos niveles plasmáticos de ácido docosahexaenoico en la $L M$, se correlacionan directamente con un mayor desarrollo cerebral y visual de los niños (10).

A pesar de las acciones de fomento en los distintos niveles de atención de salud en Chile, la prevalencia de LME para el país y la Región de la Araucanía, al sexto mes es inferior al 
60\%(11). El año 2011 se promulgó la Ley de Descanso Postnatal Parental, que aumentó 12 semanas a las 12 ya existentes (12). De esta manera, las madres pueden permanecer mayor tiempo con su hijo, favoreciendo el apego y la probabilidad de mantener la LME hasta el sexto mes. Sin embargo, la mantención de la LME o su abandono precoz depende no sólo de la extensión del descanso postnatal, sino también de otras dimensiones tales como la estructura y dinámica familiar, redes de apoyo, características socioeconómicas, planificación del retorno al trabajo, condiciones laborales, entre otras $(13,14)$. Cabe destacar en relación al contexto familiar, que el apoyo en las tareas domésticas, y las relaciones interpersonales armónicas permiten a la mujer dedicar más tiempo al bebé y al amamantamiento $(15,16)$, lo anterior se fortalece con la participación del padre en este proceso, a través del apoyo emocional y contención a la madre frente a las eventuales dificultades $(17,18)$. La falta de apoyo económico familiar en las mujeres solteras sería una de las causas que podrían llevar al destete precoz(19). Otros autores refieren que los aspectos de índole cultural como tradiciones, creencias y valores son causales que pueden facilitar o impedir el éxito de la $L M(15$, 20). Asimismo, la percepción materna respecto de las características propias del hijo, como su capacidad de succión, su necesidad de alimentación y $\operatorname{saciedad}(9,17,18)$, entre otras son algunas de las causas del destete precoz. Por esta razón conocer las experiencias de las mujeres sobre el proceso de lactancia materna permitiría revisar las prácticas de los profesionales que participan en la promoción, protección y apoyo al amamantamiento $(13,20)$.

La legislación actual, permite a las mujeres estar mayor tiempo con su hijo, modificar su vivencia y posiblemente también la dinámica familiar, al permitir la estadía de la madre en forma permanente con el hijo, lo que puede estar directamente relacionado con la mantención de la $L M$. El objetivo de esta investigación fue rescatar desde el relato de las mujeres, la identificación de los contextos que interactúan para mantener la $L M$ y explorar la percepción del beneficio que las madres le asignan a esta nueva ley.

\section{SUJETOS Y MÉTODOS}

Las participantes fueron 65 madres que amamantaron al menos un mes y que habían cumplido o se encontraban haciendo uso del descanso postnatal parental, y que controlaban a sus niños en los centros de salud familiar de la Región de la Araucanía.

Diseño cualitativo, exploratorio, descriptivo, con un enfoque desde la teoría fundamentada, que permite desarrollar y verificar teorías emergentes que permitan comprender el fenómeno en estudio, a partir de un proceso de recolección y análisis de datos sistemático, efectuando una comparación constante entre los datos y el significado de éstos.

Las madres consintieron en forma voluntaria su participación y firmaron el documento que contó con la aprobación del Comité Ético Científico de la Universidad de La Frontera (UFRO). Se excluyeron las madres que amamantaron menos de un mes y aquellas que tenían contraindicación de LM. Se planificó una muestra teórica intencionada con el fin de rescatar las experiencias de madres, según su estado civil, ocupación y edad del hijo, ya que estas variables pueden modificar la experiencia. Se realizaron cinco grupos focales con 5 a 7 participantes cada uno, 5 entrevistas en profundidad y 30 entrevistas telefónicas, siendo todas entrevistas únicas (tabla 1). Las reuniones se realizaron en salas cuna, jardines infantiles y en dependencias de la Facultad de Medicina de la UFRO; las entrevistas se efectuaron en los domicilios de las madres. La conducción de los grupos focales y las entrevistas estuvo a cargo de una investigadora experimentada en la recolección de información con estas técnicas, las madres desconocían la profesión de las investigadoras. Para las entrevistas telefónicas se utilizó una pauta tipo con preguntas predefinidas. El registro conversacional se grabó en formato $\mathrm{mp} 3$, y las notas de campo se utilizaron para complementar las transcripciones literales del discurso de las participantes. El total de entrevistas individuales y grupales fue definido una vez que se obtuvo la saturación de la información. A partir de la teoría fundamentada, se realizó un análisis de contenido inductivo de la información obtenida. Se utilizó el programa Atlas ti-6.2, para analizar el texto se efectuó una codificación abierta, y posteriormente axial. El análisis preliminar se realizó por separado, cada una de las investigadoras elevó códigos los que posteriormente se consensuaron, con estos códigos se realizó nuevamente lectura de las entrevistas, buscando similitudes y diferencias hasta obtener acuerdos entre las investigadoras. Se elaboró una matriz con los datos obtenidos de la información de las entrevistas, registrando el número de frases correspondientes a cada categoría y que dan cuenta de los aspectos positivos o negativos de la experiencia. Se establecieron macrocategorías que dan cuenta del fenómeno en estudio (tabla 2). El contar con tres estrategias de recolección de información permitió triangulación por técnica.

\section{RESULTADOS}

Los relatos de las madres fueron seleccionados según la claridad del discurso, el énfasis del mismo y las experiencias más representativas y reiteradas entre las participantes. Se identificó el tipo de entrevista: como telefónica (ET), en profundidad (EP), o grupo focal (GF).

Las madres refirieron que el aumento de la duración del postnatal genera mayor tranquilidad, dado que este tiempo de permanencia con el hijo o hija favorece el apego, el conocimiento de sus necesidades específicas, tales como el dolor, ansiedad, hambre, frío, placer, confort, entre otras.

"Excelente, me he dedicado de lleno al Agustín, ha tenido harto apego, creo que darle leche ha mejorado la relación y la complicidad entre los dos". (ET-16). "Aparte de ser cómodo, es un momento de entregar amor, cariño... reforzando el vínculo afectivo creando un lazo tremendo entre la bebé" (ET-28).

Además, valoran el postnatal actual que permite dejar al cuidado de un tercero a su hijo de seis meses, que es menos vulnerable que a los tres meses, como sucedía con el descanso postnatal anterior.

"Yo creo que es harta la diferencia porque cuando uno tiene tres meses, los ochenta y cuatro días, el bebé está muy pequeño... A veces uno no tiene la posibilidad de dejarlo con una persona más directa, que es la mamá de uno, por ejemplo, y es complicado" (GF3-1).

Entre los aspectos positivos que favorecen la lactancia materna en este período, destaca el apoyo de la pareja que se expresa en el cuidado del hijo y la participación en tareas domésticas, acciones que favorecen el amamantamiento y la compañía, seguridad y contención emocional a la madre, cuando se encuentra cansada.

“...está tan desgastada que cuando es ahí cuando uno necesita el apoyo moral, el apoyo físico, el apoyo emocional del marido...entonces, ahí estaba el esposo vigilando el mismo tomaba mi pecho se lo acomodaba el bebé y yo dormía y él era mi apoyo moral..." (GF2-4). "Mi marido me ayuda harto, él se queda con mi hijo chico cuando tengo que hacer un 
trámite, ... cuando llevo al Fernando a sus controles y en las labores de la casa" (ET - 9).

Todas las madres consideran relevante el apoyo de los integrantes de la familia para la experiencia del amamantamiento, ya que éste implica un desgaste físico y emocional.

“El apoyo de las mamás de uno es súper importante...de contención, de que te ayudan en todas las cosas, pero además de pedirles consejos" (EP - 1). "Entonces los quince días que él no está tengo el apoyo de mi mamá, de mi papá, de mi hermana para mis trámites, los mismos controles...entonces es importante la familia (GF4-1).

Entre los aspectos que no favorecen la $L M$, las madres mencionan la intranquilidad que experimentan al pensar o saber que no contarán con facilidades para el amamantamiento en su espacio laboral.
"Si, yo tuve que empezar como a los cinco meses de a poquito a ir cortándola... que empezara a tomar relleno... Porque en mis horarios yo no podía estar saliendo a cada rato para la sala cuna para ir a darle pecho" (GF4 - 2). "A pesar de que ellos me dijeron: no te preocupís, te vamos a dar todas las facilidades y toda la cuestión. Nunca. Nunca pude sacarme leche" (EP - 2). "Tengo compañeras que no le dan leche por el mismo tema que cuando vuelven a trabajar se les complica..." (ET - 18).

Además, el desconocimiento de la Ley respecto de sus derechos también genera ansiedad:

"Hasta el día de hoy me asalta esa duda... Yo tengo colegas, compañeras de trabajo ahí mismo que cumplieron un año y tres meses y la despidieron. $Y$ el derecho que es de sala cuna, y el derecho de lactancia es hasta los dos años" (GF1 - 1).

TABLA 1

Características de las participantes

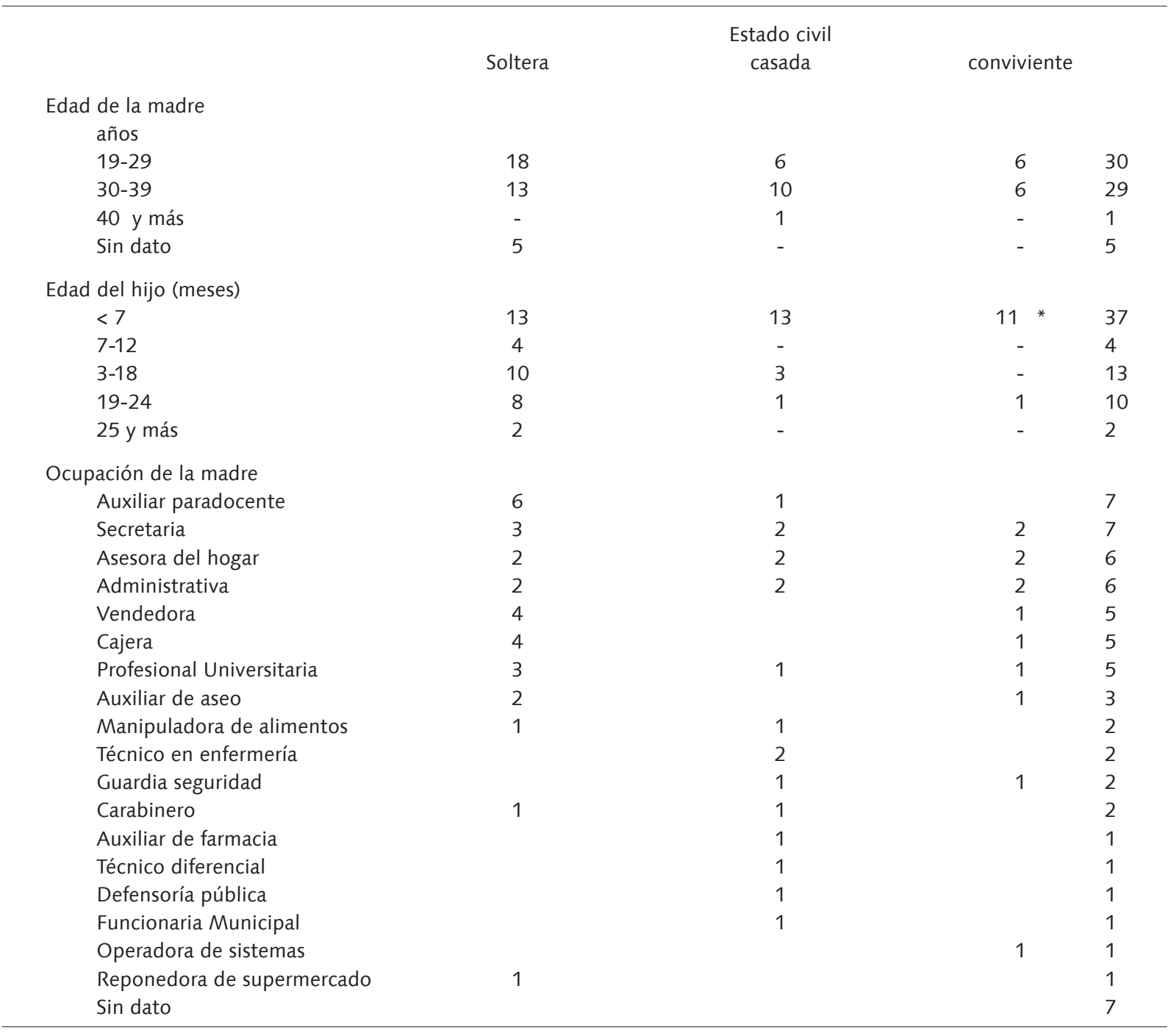

* incluye un par de gemelos 
Las madres refieren no conocer los detalles de la ley en relación con el tiempo de traslado necesario para salir a amamantar.

"Yo no lo sabía...tiene muchos vacíos legales el postnatal. Sí, porque...no hay información. (GF3 - 2). "No sé qué va a pasar después de los ochenta y cuatro días... no me he interiorizado mucho en el tema de cómo son los otros ochenta y cuatro días" (EP - 2).

Sumado al desconocimiento de la Ley, las madres expresan que la reducción del salario que algunas de ellas tuvieron fue inesperada, y que en los casos que solicitaron mayor información, no existió claridad del empleador o inconsistencias en la información recibida ya sea en la oficinas de la Comisión de Medicina Preventina e Invalidez (COMPIN), o en la inspección del trabajo.

"Efectivamente me bajó un porcentaje porque mi sueldo superaba el tope de UF máximo para ese subsidio estatal." (GF5 -6). "Me lo pasé en COMPIN. Ahora me pagaron el prenatal... ahora estamos en el proceso, que no sé si va a suceder de que me paguen el postnatal. Porque yo fui a COMPIN y me dijeron que estaba en evaluación" (ET-1).

Otro aspecto mencionado en relación al trabajo, es que otorgan permiso a la trabajadora según la conveniencia del empleador, sin relevar la prioridad de la madre:

"Yo también llegué al tema de la inspección...llegar y dejar las funciones para salir a amamantarlo, no te permiten en las empresas" (GF3-7). "Porque ellos ven siempre el bien de la empresa, a ellos les interesa que la persona trabaje ahí, cumpla sus horarios y no les interesa si el niño queda bien o no"(GF2 - 1).

El destete precoz es otro elemento que surge en el discurso de las participantes, entre las causas mencionadas está la presión familiar que reciben para iniciar aporte de fórmula, asumiendo que el lactante queda con hambre:

"La presión también es importante, muy importante... pero yo evité en toda circunstancia que le dieran relleno" (GF5 - 1). "Es como un estrés que la familia te diga no sé: tiene algo que hacer, cómprale un tarrito de algo" (GF5 - 6).

En algunos casos la indicación médica ejerce poderosa influencia para introducir fórmula, aduciendo incremento ponderal insuficiente del niño, desgaste materno o ambos.

"Porque a mí el doctor a los cinco meses y medio me dijo: tú estás muy flaca, no deberías dar pecho, estaría bueno que se lo cortes" (GF5 - 1). "Pero cuando había pasado como un mes, yo todavía no podía descansar....entonces, ahí la pediatra me dijo que no le diera, porque a lo mejor mi leche no le estaba dándole tanto alimento, porque se despertaba tanto en la noche y tenía que empezar acostumbrarse a dormir como más horas (GF1 - 3).

Otro aspecto que induce a la madre a planificar un destete precoz, es la incorporación del niño a la sala cuna al finalizar el postnatal, donde ella visualiza probables dificultades para que su hijo reciba fórmula por biberón, en los casos en que sólo ha sido amamantado:

"Entonces, la guagua tiene que empezar a no comer a los seis, sino a los cinco, ¿para qué?...para que nosotras estemos seguras de que cuando llegue a la Sala Cuna va a recibir leche, comida. Lo mismo pasa con la mamadera, hay que ir preparando antes" (GF2 - 2).

Todas las madres mencionan valorar la lactancia materna y que esta es una experiencia satisfactoria y placentera:

"Nada más, ni siquiera agua. Solamente pecho porque ahí están los nutrientes, las defensas. Entonces yo me quedo con eso...ella no se ha enfermado nunca, ni siquiera así un resfria-

\section{TABLA 2}

Categorías emergentes y distribución según valoración positiva o negativa de la experiencia en lactancia materna.

\begin{tabular}{|c|c|c|c|}
\hline Macro-categoría & Categoría & $\mathrm{N}^{\circ}$ Relatos positivos & $\mathrm{N}^{\circ}$ Relatos negativos \\
\hline \multirow[t]{3}{*}{ Familia } & apoyo familiar & 52 & \\
\hline & presión familiar & & 2 \\
\hline & falta de red familiar & & 2 \\
\hline \multirow[t]{2}{*}{ Pareja } & Apoyo de pareja & 30 & \\
\hline & Falta de apoyo de la pareja & & 9 \\
\hline Otras personas cercanas & apoyo & 3 & \\
\hline \multirow{4}{*}{ Amamantamiento } & Placer de amamantar & 55 & \\
\hline & Valoración de la leche materna & 46 & \\
\hline & problemas de amamantamiento & & 16 \\
\hline & amamantamiento satisfactorio & 43 & \\
\hline \multirow[t]{4}{*}{ Valoración de postnatal } & Valoración de postnatal & 57 & \\
\hline & Postnatal escaso & & 22 \\
\hline & Apego & 47 & \\
\hline & Tranquilidad & 30 & \\
\hline \multirow[t]{2}{*}{ Control pediátrico } & indicación médica destete precoz & & 5 \\
\hline & pediatra apoyador & 4 & \\
\hline \multirow[t]{7}{*}{ Aspectos laborales } & reducción salario & & 10 \\
\hline & desconocimiento de derechos laborales & & 3 \\
\hline & hora de amamantamiento insuficiente & & 32 \\
\hline & Falta de acondicionamiento laboral & & 12 \\
\hline & apoyo laboral & 2 & \\
\hline & conocimiento del postnatal & 2 & \\
\hline & Angustia & & 4 \\
\hline
\end{tabular}


do... entonces uno le va entregando las defensas en el pecho" $(E P-4)$. "Bien, es una responsabilidad y una experiencia muy bonita... estar con el hijo te cambia la vida, ver de otra forma como llevar la vida adelante" (ET - 26).

Sin embargo, la mayoría de ellas manifiestan dolor como problema durante el primer mes de amamantamiento, el cual se resuelve positivamente.

"Los primeros meses sufrí, porque me dolían demasiado,....me hacía heridas, y yo no tenía tanto pezón. (GF1-3). "Si. Me costó mucho, me dolían mucho los pezones y se me hinchaban y todo" (GF4 -2). "Es doloroso al principio y, porque después el pezón ya tomó su forma que es lo que el niño requiere para poder alimentarse. Es rico sentir que las mamas se llenan con leche y que el hijo te saque, se alimenta de ti, ¡Es súper rico!" (GF1 - 4).

Otro aspecto mencionado por las madres dice relación con el apoyo que reciben de los profesionales de la salud para promover la mantención de la leche materna:

"La matrona se encargó,... ella me enseñó a mí a sacarme manualmente, y también le enseñó a mi esposo" (GF1 - 4). "recibí ayuda acá en la casa, vinieron dos matrones y me enseñaron a extraerme la leche y como almacenarla, era como una clínica de lactancia" (ET-5). "En el consultorio en el control con la nutricionista ahí me enseñaron todo" (ET - 29).

Algunas madres manifestaron la experiencia de estar sin apoyo familiar o de la pareja durante el período del amamantamiento, lo que significó una carga emocional importante, desgaste físico, y como consecuencia de este entorno desfavorable, dificultades en el amamantamiento o en la mantención de la producción láctea.

"No, en mi caso desde que nació no lo quiso (la pareja)... igual tuve una mastitis" (GF4 - 2). "Yo siempre he estado sola, ahora yo vivo sola, sola con mi hijo y ha sido difícil, es complicado hasta el día de hoy" (GF3 - 3).

Las madres valoran la extensión del tiempo del postnatal, sin embargo, lo consideran insuficiente, dado que el niño a los cinco meses y medio, no está en condiciones de recibir alimento sólido, lo que acelera la introducción de fórmula láctea.

"Obviamente haberlo aumentado de los tres meses a los seis meses. Sí, por supuesto fue mejor, pero sigue siendo insuficiente." (GF1 - 1)

Otro aspecto mencionado que explicaría las dificultades en mantener la $L M$ cuando la madre se reintegra al trabajo, es el desconocimiento de la técnica de extracción y conservación láctea, información que no todas las madres reciben de los profesionales de salud, buscando respuestas en otras fuentes.

"Yo lo intenté, yo me compré un saca leche, pero no pude, después le dí mamadera no más" (ET - 1). "Una prima me enseñó cómo se hacía" (ETP - 4). "Yo tengo que reconocer que fue mi hermana mayor" (ET - 4). "Internet" (ET - 2). "Tutoriales que habían en youtube, google y ahí aprendí..." (ET - 20).

Destaca que tres entrevistadas, profesionales universitarias manifestaron sentir necesidad de regresar al trabajo y reincorporarse a sus actividades:

"Si, si, ojalá (estuviera recibiendo alimento sólido el hijo) porque uno igual va necesitando como su vida o como otras cosas. Como que una está acostumbrada a trabajar" (EP - 1). "Yo estaba loca en la casa, yo quería sólo volver a trabajar..." (GF5 - 6).

\section{DISCUSIÓN Y CONCLUSIONES}

En este grupo de madres, el postnatal extendido permite la mantención de la $L M$, pero principalmente se constituye en un factor que fortalece el vínculo ente la madre y su hijo, siendo un período que le permite reconocer conductas, necesidades propias del niño y lograr un amamantamiento satisfactorio. El apoyo familiar y principalmente el de la pareja cumple un papel determinante en la contención emocional y en el cuidado de los hijos, que le otorga seguridad y tranquilidad, favoreciendo de esta forma, la producción láctea y el amamantamiento. Estos hallazgos son similares a los descritos en la Encuesta Nacional de Lactancia Materna (ENALMA), y también mencionados por otros autores(16,17, 21-26)

Similar a otros estudios $(23,27,28)$, durante el primer mes de vida del hijo, es posible que las madres refieran dolor para amamantar, presencia de grietas del pezón o ambas, factores que se asocian con la discontinuidad de la lactancia materna (29). En esta etapa es fundamental la intervención que realiza el profesional de la salud, para favorecer la $L M$ y postergar la introducción de fórmula láctea. Sin embargo, las madres mencionan, al igual que otro autores $(23,25)$, que la educación brindada por los profesionales de salud tienen menor impacto que el generado por el apoyo de algún miembro de la familia para la mantención de la LME.

El reingreso al trabajo es una de las razones referidas por las madres para inducir el destete precoz, al igual que lo reportado por otros autores $(13,16,19,24)$; por tal motivo, y cuando la madre no puede dejar a otro familiar al cuidado de su hijo, planifica el ingreso del niño a la sala cuna, situación que se asocia con la introducción de fórmula láctea antes de los seis meses de vida y el consecuente abandono de la LME.

Cabe mencionar que en este estudio no se obtuvo el relato de madres que al momento de ingresar al trabajo dejan al cuidado de su hijo a algún familiar u otra persona de confianza. Esta limitante no permitió indagar si en este contexto las madres también introducen en forma precoz una fórmula láctea. Además, es probable que las madres de hijos mayores de seis meses que participaron de las entrevistas, sólo tuvieran recuerdos positivos o negativos que impactaron en su experiencia, no aportando con información completa, que permita comprender el fenómeno en su totalidad.

Otro aspecto relacionado al reingreso laboral es el desconocimiento de la madre y también de algunos empleadores, respecto de los derechos legales que protegen el tiempo destinado al desplazamiento y al amamantamiento del hijo. Esta desinformación genera estrés e intranquilidad que dificulta la mantención de la $\operatorname{LME}(18,23)$. La difusión de estos derechos laborales es fundamental para apoyar a las trabajadoras que desean mantener la LME por un período mayor a los seis meses.

A pesar que el aprendizaje de la extracción láctea, las condiciones, tiempo y lugar de la conservación son relevantes para la mantención de la LME (13), no todas las madres reciben información al respecto. Algunas de ellas obtienen la información de familiares, desde internet y en menor proporción de profesionales de la salud. Al igual que en otro estudio (23), se plantea como una estrategia para favorecer el mantenimiento de la $L M E$, educar en la extracción y conservación de ésta.

Resulta fundamental y significativo el acompañamiento permanente de la pareja y la familia para prolongar el amamantamiento, por esta razón se propone que el equipo de salud pueda identificar dentro del núcleo familiar a la persona que acompañará a la madre en este período crítico de la lactancia, y por otro lado, asegurar el conocimiento del manejo de la leche materna extraída para favorecer la práctica en todas las madres trabajadoras, y especialmente a las que dejan a sus hijos en sala cuna, distantes de su lugar de trabajo. 


\section{RESUMEN}

La prevalencia de lactancia materna exclusiva hasta el sexto mes de edad en Chile no supera el 60\%, a pesar de la promulgación de la Ley de Descanso Postnatal Parental el año 2011, la cual permite a las madres permanecer con el recién nacido hasta las veinticuatro semanas de vida. El objetivo de este trabajo fue explorar, desde la perspectiva de las madres, cuales son los aspectos involucrados en la duración del periodo de lactancia materna exclusiva. Sujetos y Métodos: Exploración cualitativa desde la teoría fundamentada. Se realizaron entrevistas semiestructuradas a 35 participantes y cinco grupos focales con 30, en total 65 madres trabajadoras participaron en el estudio. Se realizó un análisis de contenido inductivo, utilizando el software atlas ti-6.2. Los criterios de validez incluyeron triangulación por investigador y por técnica. Resultados: Los elementos identificados como favorecedores de la lactancia materna son la duración del período del descanso postnatal, el apoyo de la pareja, de la familia y de los profesionales de salud. Los que representan una barrera son la presencia de dolor al amamantar y la decisión de destetar precozmente, la cual puede estar influenciada por familiares o profesionales de la salud. Conclusiones: Dado la fuerte dependencia de factores como la pareja y la familia en la mantención de la lactancia materna, se propone que los profesionales de salud involucrados consideren estos aspectos en la promoción.

Palabras clave: Lactancia materna; estudio cualitativo; apoyo social; promoción de la salud; profesionales de la salud.

Agradecimientos: A las madres que compartieron su experiencia.

Este estudio fue financiado por el Proyecto DIUFRO $N^{\circ} 14-0018$

\section{BIBLIOGRAFÍA}

1. Schellhorn $C$, Valdés $V$, Juez $G$, Niño $R$, Weason $R$, Mena $P$, et al. Maternal Breastfiding Manual [Internet]. 2010. 238 p. Available from: http://scholar.google.com/scholar?hl=e $n \& b t n G=$ Search\&q=intitle:Lactancia+Materna\#O\nhttp:// books.goog/e.com/books?hl=en\&/r=\&id=Ulxyj72VZDOC\&o $i=$ fnd\&pg $=P R 9 \& d q=$ Manual+de+Lactancia+Materna\&ots= jpkU9wqOvV\&sig=gwfmLOpWDozwOdCfQK9SE4QU2_E

2. Anderson A, Burggren A. Cognitive and neurodevelpmental benefits of extended formula-feeding in infants: Re: Deoni et al.2013. Neurolmage. 2014; 100(3): 706-709.

3. Brion M-J, Lawlor D, Matijasevich A, Horta B, Anselmi $L$, Araújo $C L$, et al. What are the causal effects of breastfeeding on IQ, obesity and blood pressure? Evidence from comparing high-income with middle-income cohorts. Int J Epidemiol [Internet]. 2011 Jun [cited 2013 Oct 30];40(3):670-80. Available from: http://www.pubmedcentral.nih.gov/articlerender.fcgi?artid $=3147072 \&$ tool $=p$ mcentrez\&rendertype=abstract.

4. Huang J, Peters K, Vaughn M, Witko C. Breastfeeding and trajectories of Children's cognitive development. Dev Sci. 2014 May;17(3):452-461.

5. Jedrychowski W, Perera F, Jankowsky J, Butscher M, Mroz E, Flak $E$, et al. Effect of Effect of Exclusive Breastfeeding on the Development of Children 's Cognitive Function in the Krakow prospective birth cohort study. Eur j Pediatr. 2012;17(1):151-8.

6. Kramer M. "Breast is best": The evidence. Early Hum Dev [Internet]. Elsevier Ltd; 2010 Nov [cited 2013 Oct 30];86(11):729-32. Available from: http://www.ncbi. nlm.nih.gov/pubmed/20846797

7. Patel R, Oken E, Bogdanovich N, Matush L, Sevkovskaya $Z$, Chalmers B, et al. Cohort profile: The promotion of breastfeeding intervention trial (PROBIT). Int J Epidemiol. 2014;43(3):679-90.

8. Pinto L. Wonders and magic of human neurodevelopment. Rev Chil Pediatr 2008;79(1):18-20.

9. Papp L. HHS Public Access. Child care Heal. 2014; 40(5): 740-6.

10. Valenzuela $R$, Morales J, Sanhueza J. VB. Docosahexaenoic acid (DHA), an essential fatty acid at the brain. Rev Chil Nutr. 2013;40(4):383-90.

11. Minsal. National survey on maternal breastfeeding in primary care. Access in November 9th, 2015 in http:// web.minsal.cl/sites/default/files/INFORME_FINAL_ENALMA_2013.pdf

12. Article $n^{\circ} 197$, Labour Code, Law $n^{\circ} 20545$. Modifies norms on maternity and incorporates paternal postnatal

13. Navarro-Estrella M, Duque-López MX, Trejo J, Pérez J. Factors associated with short duration of breastfeeding in Mexican working women. Salud Pública Mex. 2003;45(4):276-84.

14. Turnbull B, Escalante-Izeta $E$, Klunder M. The role of social networks in exclusive breastfeeding. Rev Med Inst Mex Seguro Soc. 2006;44(2):97-104.

15. Niño $R$, Silva $G$, Atalah E. Determinants of exclusive breastfeeding in health centers in Santiago, Chile. Rev Chil Pediatr. 2012;83(2):161-9.

16. Becerra-Bulla F, Rocha-Calderón L, Fonseca-Silva D, Bermúdez-Gordillo L. Net D, Database C. The family and social environment of the mother as a factor that promotes or hinders breastfeeding. Rev Fac Med. 2015:63(2):217-27.

17. Belintxon-Martín M, Zaragüeta M, Adrián M, LópezDicastillo O. Initiating breastfeeding: experiences of firsttime mothers. An Sist Sanit Navar. 2011;34(3):409-18.

18. Moore E, Coty M-B. Prenatal and postpartum focus groups with primiparas: Breastfeeding attitudes, support, barriers, self-efficacy, and intention. J Pediatr Heal Care. 2006;20(1):35-46.

19. Pino JL, López M, Medel A. OA. Factors affecting the duration of exclusiv breastfeeding in a rural community of Chile. Rev Chil Nutr [Internet]. 2013;40(1):48-54. Available from: http://www.scielo.cl/pdf/rchnut/v40n1/art08.pdf

20. Cerda L. Breast feeding and care management. Rev Cubana Enferm. 2011;27(4):327-36.

21. Cardenas $M H$, Montes $E$, Varon $M$, Arenas $N$, Reina $R$. Bio-psychosocial profile of the mother and her relation with the abandonment of exclusive maternal lactation. Enferm. Glob. 2010;20:1-10.

22. Gorrita R, Ravelo Y, Ruiz E. Brito B. Desires, aptitudes and knowledge about breastfeeding in women at their third trimester of pregnancy. Rev Cubana Pediatr. 2012;84(2):165-75.

23. Gamboa E, Lopez N, Prada GE, Gallo K. Knowledge, attitudes and practices related to breast-feeding in women in reproductive age in a vulnerable population. Rev Chil Nutr. 2008;35(1):1-17.

24. Müller F, Silva IA. Social representations about support for breastfeeding in a group of breastfeeding women. Rev Lat Am Enfermagem. 2009;17(5):651-7.

25. Losa-Iglesias M, Rodriguez-Vasquez $R$, Becerro de Bengoa $R$. The Grandmother's Role in Breastfeeding. Aquichán (Colombia). 2013;13:270-9.

26. Tsai S-Y. Influence of Partner Support on an Employed 
Mother's Intention to Breastfeed After Returning to Work. Breastfeed Med [Internet]. 2014;9(4):222-30. Available from: http://www.ncbi.n/m.nih.gov/pubmed/24650363

27. Cardalda EB, Martínez J, Alcalá A, Talavera J, Hernández $S$, Dávila M, et al. Enabling factors and difficulties for successful breastfeeding in different occupational groups of Puerto Rican mothers. (Spanish). Puerto Rican J Psychol / Rev Puertorriqueña Psicol [Internet]. 2012;23(2):91-108. Available from: http://flagship.luc.edu/login?url=http:// search.ebscohost.com/login.aspx?direct $=$ true $\& d b=a 9 h \& A$
$N=79934908$ \&site $=$ ehost-live

28. Prieto $R$, Baeza B. Breastfeeding: Prevalence of nipple cracking and pain in breastfeeding mothers in the Araucanía. Rev Colomb Obstet Ginecol. 2013;64(3):229-33.

29. Vieira T, Vieira G, De Oliveira N, Mendes CMC, Giugliani $E$, Silva L. Duration of exclusive breastfeeding in a Brazilian population: new determinants in a cohort study. BMC Pregnancy Childbirth [Internet]. 2014;14(1):175. Available from: http://www.biomedcentral.com/14712393/14/175. 